Bull. Austral. Math. Soc.

Vol. 50 (1994) [299-312]

\title{
SCHRÖDINGER OPERATORS WITH MAGNETIC AND ELECTRIC POTENTIALS
}

\section{YU KAIQI}

In the present paper, we consider Schrödinger operators which are formally given by $P=-\sum_{j=1}^{N}\left(\partial_{j}-i a_{j}\right)^{2}+V$ in $L^{2}\left(R^{N}\right)$. In Section 2 and 3 we prove that $P$ has a regularly accretive extension which is a self-edjoint extension of $P$ and it is the only self-adjoint realisation of $P$ in $L^{2}\left(R^{N}\right)$ when $\vec{a}$ satisfies $\vec{a}=\left(a_{1}, a_{2}, \cdots, a_{N}\right) \in$ $L_{\text {loc }}^{2}\left(R^{N}\right)^{N}, a_{j}$ real-valued, $1 \leqslant j \leqslant N, V \in L_{\text {loc }}^{1}\left(R^{N}\right)$, real-valued and the negative part $V_{-}:=\max (0,-V)$ satisfys $\int_{R^{N}} V_{-}|\varphi|^{2} d x \leqslant C_{1}\|\nabla \varphi\|^{2}+C_{2}\|\varphi\|^{2}$ $\varphi \in H^{1,2}\left(R^{N}\right)$, with constants $0 \leqslant C_{1}<1, C_{2} \geqslant 0$ independent of $V$. In Section 4, we prove that $P$ is essential self-adjoint on $C_{0}^{\infty}\left(R^{N}\right)$ when $\vec{a}, V$ satisfy $\vec{a} \in L_{\mathrm{loc}}^{4}\left(R^{N}\right)^{N}, \operatorname{div} \vec{a} \in L_{\mathrm{loc}}^{2}\left(R^{N}\right) ; V=V_{1}+V_{2}, V$ real-valued, $V_{i} \in L_{\mathrm{loc}}^{2}\left(R^{N}\right)$, $i=1,2, V_{1}(x) \geqslant-C|x|^{2}$, for $x \in R^{N}$ with $C \geqslant 0$ and $0 \geqslant V_{2} \in K_{N}$.

\section{INTRODUCTION}

In the present paper, we consider Schrödinger operators which are formally given by $P=-\sum_{j=1}^{N}\left(\partial_{j}-i a_{j}\right)^{2}+V$, where $V$ is an electric potential and $\vec{a}=\left(a_{1}, a_{2}, \cdots, a_{N}\right)$ is a singular magnetic vector potential. In solid state physics, this corresponds to a simple one-electron model of a crystal in a magnetic field, the (short-range) potential $V$ describing impurities of the crystal (Reed and Simon [5, Vol.IV, Section VII.16]).

Schrödinger operators with magnetic vector potentials have been studied extensively (Leinfelder and Simader [4], Simon [9], Simader [7], Hinz and Stolz [2] and the references given therein). In Section 2 and 3, we make the general assumption $\mathrm{s}$

$$
\begin{aligned}
\vec{a}=\left(a_{1}, a_{2}, \cdots, a_{N}\right) & \in L_{\text {loc }}^{2}\left(R^{N}\right)^{N}, a_{j} \text { real-valued, } 1 \leqslant j \leqslant N, \\
V & \in L_{\text {loc }}^{1}\left(R^{N}\right), \text { real-valued, }
\end{aligned}
$$

the negative part $V_{-}:=\max (0,-V)$ satisfying

$$
\begin{aligned}
& \int_{R^{N}} V_{-}|\varphi|^{2} d x \leqslant C_{1}\|\nabla \varphi\|^{2}+C_{2}\|\varphi\|^{2}, \varphi \in H^{1,2}\left(R^{N}\right) \\
& \text { with constants } 0 \leqslant C_{1}<1, C_{2} \geqslant 0 \text { independent of } V .
\end{aligned}
$$

Received 16 November 1993

Copyright Clearance Centre, Inc. Serial-fee code: 0004-9729/94 \$A2.00+0.00. 
Since $C_{0}^{\infty}\left(R^{N}\right)$ is dense in $H^{1,2}\left(R^{N}\right)$ and $V_{-}^{1 / 2}$ is a closed operator from $L^{2}\left(R^{N}\right)$ into $L^{2}\left(R^{N}\right)$ as a multiplication operator, (1.3) can be written

$$
\int_{R^{N}} V_{-}|\varphi|^{2} d x \leqslant C_{1}\|\nabla \mid \varphi\|^{2}+C_{2}\|\varphi\|^{2}, \quad \varphi \in B^{1,2}\left(R^{N}\right)
$$

Condition (1.3) or (1.3)' is for example satisfied if $V_{-} \in K_{N}$ (in fact, $C_{1}$ may be zero in this case), where

$$
\begin{gathered}
K_{N}=\left\{V \in L_{\mathrm{loc}}^{2}\left(R^{N}\right): \lim _{t \downarrow 0} \omega_{N, t}(V)=0\right\}, \\
\omega_{N, t}(V)=\sup _{x \in R^{N}} \int_{|x-y|<t}|V(y)||x-y|^{2-N} d y, \text { for } t>0 \text { and } N \geqslant 3 .
\end{gathered}
$$

For $N=2,|x-y|^{2-N}$ has to be replaced by $\log |x-y|^{-1}$ in Equation (1.5); for $N=1, K_{N}$ coincides with $L_{\text {loc }}^{1}(R)$ (compare [1] for these definitions).

Now we define a sesquilinear form $h_{\vec{a}, V}$ in the Hilbert space $L^{2}\left(R^{N}\right)$ by

$$
h_{\vec{a}, V}(u, v)=\sum_{j=1}^{N}\left(\left(\partial_{j}-i a_{j}\right) u,\left(\partial_{j}-i a_{j}\right) v\right)+\int_{R^{N}} V u \bar{v} d x
$$

for $u, v$ from

$$
\begin{aligned}
D\left(h_{\vec{a}, V}\right)=\left\{u \in L^{2}\left(R^{N}\right):\left(\partial_{j}-i a_{j}\right) u \in L^{2}\left(R^{N}\right)\right. & \\
& \left.1 \leqslant j \leqslant N, V|u|^{2} \in L^{1}\left(R^{N}\right)\right\},
\end{aligned}
$$

where $\left(\partial_{j}-i a_{j}\right) u$ is defined in the sense of distribution. $h_{\vec{a}, v}$ is symmetric semibounded, densely defined and closed, this is shown in [4] for $V \geqslant 0$. To accommodate $V_{-}$, it is important to note that (compare [4, Equation (3.6)])

$$
\partial_{j}|u| \leqslant\left|\left(\partial_{j}-i a_{j}\right) u\right| \quad u \in D\left(h_{\vec{a}, v_{+}}\right)
$$

where $V_{+}=V+V_{-}$. Hence, if (1.3) holds, $V_{-}$has relative form bound $C_{1}<1$ with respect to $h_{\vec{a}, V_{+}}$and [3, Theorem VI-1.33] applies.

Let $B_{\vec{a}, V}$ denote the self-adjoint and semibounded operator associated with $h_{\vec{a}, V}$ by [3, Theorem VI-2.1]. Instead of $H_{0, V}$, we write $-\Delta+V$. Then $H_{\vec{a}, V}$ is a self-adjoint realisation of $P$ in $L^{2}\left(R^{N}\right)$ in the sense of form and $D\left(H_{\vec{a}, V}\right)=\left\{u \in L^{2}\left(R^{N}\right)\right.$ : 
$\left.\left(\partial_{j}-i a_{j}\right) u \in L^{2}\left(R^{N}\right),|V|^{1 / 2} u \in L^{2}\left(R^{N}\right), P u \in L^{2}\left(R^{N}\right)\right\}$, where $P$ acts on $u$ in the distribution sense.

In Section 2, we consider the regularly accretive extension of $P$, which is also a self-adjoint extension of $P$. We point out that when $\vec{a}, V$ satisfy (1.1)-(1.3), $P$ has a regularly accretive extension $F_{\vec{a}, V}$ and $F_{\vec{a}, V}=H_{\vec{a}, V}$ (Theorem 2.2 and Theorem 2.3). In Section 3 we prove that one can define a maximal self-adjoint realisation $\widetilde{\boldsymbol{H}}_{\vec{a}, V}$ of $P$ in $L^{2}\left(R^{N}\right)$ as follows:

$$
\begin{gathered}
D\left(\widetilde{H}_{\vec{a}, V}\right)=\left\{u \in L^{2}\left(R^{N}\right):\left(\partial_{j}-i a_{j}\right) u \in L_{\mathrm{loc}}^{2}\left(R^{N}\right),\right. \\
\left.\qquad|V|^{1 / 2} u \in L_{\mathrm{loc}}^{2}\left(R^{N}\right), P u \in L^{2}\left(R^{N}\right)\right\}, \\
\widetilde{H}_{\vec{a}, V} u=P u, \quad u \in D\left(\widetilde{H}_{\vec{a}, V}\right) .
\end{gathered}
$$

It is clear that $\widetilde{H}_{\vec{a}, V}$ is an extension of $H_{\vec{a}, V}$. In fact, we have $\widetilde{B}_{\vec{a}, V}=H_{\vec{a}, V}$ (Theorem 3.1). In [7], Simader considered a Schrödinger operator $T u=-\Delta u+V u$ on $D(T)=C_{0}^{\infty}\left(R^{N}\right)$ when the potential $V$ satisfies

(H) $\left\{\begin{array}{l}V=V_{1}+V_{2}, V \text { real-valued, } V_{i} \in L_{\text {loc }}^{2}\left(R^{N}\right), \quad i=1,2, \\ V_{1}(x) \geqslant-C|x|^{2} \text { for } x \in R^{N} \text { with suitable constant } C \geqslant 0 \text { and } 0 \geqslant V_{2} \in K_{N} .\end{array}\right.$

He proved that $T$ is essentially self-adjoint when $V$ satisfies $(\mathrm{H})$ and $V_{1} \geqslant 0$, see [7, Theorem 2]. In Section 4, we consider the self-adjoint realisation of $P$ in $L_{\text {loc }}^{2}\left(R^{N}\right)$ in the sense of operator when $V$ satisfies (H) and $\vec{a} \in L_{\text {loc }}^{4}\left(R^{N}\right)^{N}$., $\operatorname{div} \vec{a} \in L_{\text {loc }}^{2}\left(R^{N}\right)$. We prove that $P$ is essential self-adjoint on $C_{0}^{\infty}\left(R^{N}\right)$. Here, we must point out that Simader's proof of the above theorem is completely dependent on the local boundedness result in [8], but this method fails to be used in our case since $-(\nabla-i \vec{a})^{2}+V$ is not a real differential operator on $C_{0}^{\infty}\left(R^{N}\right)$. We avoid the estimation of local boundedness by means of the self-adjoint realisation $H_{\vec{a}, V}$ of $P$ in the sense of form. Recently, Hinz and Stolz proved that when $\vec{a} \in L_{\text {loc }}^{4}\left(R^{N}\right)^{N}$, div $\vec{a} \in L_{\text {loc }}^{2}\left(R^{N}\right), V \in L_{\text {loc }}^{2}\left(R^{N}\right)$ and $V_{-} \in K_{N}+O\left(|x|^{2}\right), P$ is essential self-adjoint on $C_{0}^{\infty}\left(R^{N}\right)$. Their methods are the same as Simader's.

\section{THE REgUlaRLY ACCRETIVE EXTENSION OF $P$}

Let $\boldsymbol{H}$ denote a complex Hilbert space with inner product $(u, v)_{H}$ and norm $\|u\|_{H}=(u, u)_{H}^{1 / 2}$. We suppose that there is a dense subspace $W$ of $H$ which is a Hilbert space with inner product $(u, v)_{W}$ and norm $\|u\|_{W}=(u, u)_{W}^{1 / 2}$ with $u \in W$. 
Suppose the identity map $W \rightarrow H$ is a bounded operator, that is, there is a constant $K_{0}$ such that for all $u \in W$,

$$
\|u\|_{H} \leqslant K_{0}\|u\|_{W}
$$

Suppose further there is a bilinear form $b(u, v)$ defined on $W \times W$ with values in $\mathbb{C}$ and a constant $K_{1}$ such that for all $u, v$ in $W$,

$$
|b(u, v)| \leqslant K_{1}\|u\|_{W}\|v\|_{W} .
$$

We may define the linear operator associated with $b$ to be that operator $A$ with domin $D(A) \subseteq W$ such that $u \in D(A)$ and $A u=v$ if and only if $b(u, w)=(v, w)$ for all $w \in W$.

We now make the fundamental

Definition: A linear operator $A$ is said to be regularly accretive if it is associated with a bilinear form $b$ which in addition to satisfying (2.2), also satisfies

$$
\|u\|_{W}^{2} \leqslant K_{2}\left(\operatorname{Re} b(u, u)+K_{3}\|u\|_{H}^{2}\right)
$$

for all $u$ in $W$ and fixed constants $K_{2}$ and $K_{3}$.

It can be shown that a regularly accretive operator is densely defined and closed. In addition its spectrum is contained in some half-space $\operatorname{Re} \lambda>K$ of the complex-plane. If $b$ is symmetric, then $A$ is a semibounded self-adjoint operator.

Now suppose $A_{0}$ is a linear operator in $H$ whose domain $D\left(A_{0}\right)$ is not necessarily dense in $\boldsymbol{H}$. The following lemma will be useful for us.

Lemma 2.1. Let $U$ be a dense subspace of $W$ which contains $D\left(A_{0}\right)$. Suppose $b(\cdot, \cdot)$ is a bilinear form on $U \times U$ which satisfies inequalities (2.1), (2.2) and (2.3) for all $u$ and $v$ in $U$. If

$$
b(u, v)=\left(A_{0} u, v\right)_{H}
$$

for all $u$ in $D\left(A_{0}\right)$ and $v$ in $U$, it follows that $A_{0}$ has a regularly accretive extension A.

The proof follows directly from the observation that the inequalities $(2.1),(2.2)$ and (2.3) as well as the form $b$ itself extend to all of $W$ by continuity. That the regularly accretive operator $A$ associated with $b$ and $W$ is an extension of $A_{0}$ follows from (2.4).

A fuller account of the ideas here can be found in Schechter [6] and Kato [3]. 
In the sequel, we consider the regularly accretive extension of $P$. Here, suppose $\vec{a}, V$ satisfy (1.1)-(.13). Define the operator $F_{0}$ on $C_{0}^{\infty}\left(R^{N}\right)$ as follows:

$$
\begin{gathered}
D\left(F_{0}\right)=\left\{u \in C_{0}^{\infty}\left(R^{N}\right):|V|^{1 / 2} u \in L^{2}\left(R^{N}\right), P u \in L^{2}\left(R^{N}\right)\right\} \\
F_{0} u=P u, \quad u \in D\left(F_{0}\right),
\end{gathered}
$$

where $P$ acts on $u$ in the distribution sense.

Obviously, $F_{0}$ is a linear operator in $L^{2}\left(R^{N}\right)$.

TheOREM 2.2. Let $\vec{a}, V$ and $F_{0}$ as above, then $F_{0}$ has a regularly accretive extension $F_{\vec{a}, v}$.

Proof: Let $U$ denote the space $C_{0}^{\infty}\left(R^{N}\right)$ and let $W$ be the closure of $U$ with respect to the norm

$$
\|u\|_{W}=\left(\int_{R^{N}}\left(\sum_{j=1}^{N}\left|\left(\partial_{j}-i a_{j}\right) u\right|^{2}+V_{+}|u|^{2}\right) d x+\|u\|^{2}\right)^{1 / 2}
$$

where $V_{+}=\max (0, V)$.

By (1.3) and (1.8), we can easily deduce

$$
\int_{R^{N}} V_{-}|u|^{2} d x \leqslant C_{1} \sum_{j=1}^{N}\left\|\left(\partial_{j}-i a_{j}\right) u\right\|^{2}+C_{2}\|u\|^{2}
$$

for all $u$ in $W$.

Further we define a bilinear form $b$ on $W \times W$ by the equation

$$
b(u, v)=\int_{R^{N}} V u \bar{v} d x+\sum_{j=1}^{N}\left(\left(\partial_{j}-i a_{j}\right) u,\left(\partial_{j}-i a_{j}\right) v\right) .
$$

Then for all $u$ in $D\left(F_{0}\right)$ and $v \in U, b(u, v)=\left(F_{0} u, v\right)$ and (2.1) is clear from (2.5). We see by Lemma 2.1 that we need only verify inequalities (2.2) and (2.3), that is, we need to find three positive constants $K_{1}, K_{2}$ and $K_{3}$ such that for all $u, v$ in $W$,

$$
\begin{gathered}
|b(u, v)| \leqslant K_{1}\|u\|_{W} \cdot\|v\|_{W} \\
\|u\|_{W}^{2} \leqslant K_{2}\left(b(u, u)+K_{3}\|u\|^{2}\right) .
\end{gathered}
$$


In fact,

$$
\begin{aligned}
&|b(u, u)| \leqslant \int_{R^{N}}\left(\sum_{j=1}^{N}\left|\left(\partial_{j}-i a_{j}\right) u\right|^{2}+|V||u|^{2}\right) d x \\
& \leqslant \int_{R^{N}}\left(\sum_{j=1}^{N}\left|\left(\partial_{j}-i a_{j}\right) u\right|^{2}+V_{+}|u|^{2}\right) d x \\
&+C_{1} \int_{R^{N}} \sum_{j=1}^{N}\left|\left(\partial_{j}-i a_{j}\right) u\right|^{2} d x+C_{2}\|u\|^{2} \\
& \leqslant\left(1+C_{1}\right) \int_{R^{N}}\left(\sum_{j=1}^{N}\left|\left(\partial_{j}-i a_{j}\right) u\right|^{2}+V_{+}|u|^{2}\right) d x+C_{2}\|u\|^{2}
\end{aligned}
$$

Thus, there is a constant $K_{1}>0$ such that (2.7) holds. Also,

$$
b(u, u)=\|u\|_{W}^{2}-\int_{R^{N}} V_{-}|u|^{2} d x-\|u\|^{2},
$$

so we have

$$
\begin{aligned}
\|u\|_{W}^{2} & =b(u, u)+\int_{R^{N}} V_{-}|u|^{2} d x+\|u\|^{2} \\
& \leqslant b(u, u)+C_{1}\|u\|_{W}^{2}+\left(1+C_{2}\right)\|u\|^{2} .
\end{aligned}
$$

Since $0 \leqslant C_{1}<1$, there exist $K_{2}, K_{3}>0$ such that (2.8) holds. By Lemma 2.1, $F_{0}$ has a regularly accretive extension $F_{a, v}$ in $L^{2}\left(R^{N}\right)$.

Obviously, $F_{\vec{a}, v}$ is also a self-adjoint extension in the sense of form. What is the connection between $F_{\vec{a}, v}$ and $H_{\vec{a}, V}$ ? The following result answers this question.

THEOREM 2.3. $F_{\vec{a}, V}=H_{\vec{a}, V}$.

Proof: From the proof of Theorem 2.2, we have

$$
\left(H_{\vec{a}, V} u, v\right)=\int_{R^{N}}\left(\sum_{j=1}^{N}\left(\partial_{j}-i a_{j}\right) u \cdot \overline{\left(\partial_{j}-i a_{j}\right) v}+V u \bar{v}\right) d x
$$

for $u \in D\left(H_{\vec{a}, v}\right), v \in W$.

Since $F_{\vec{a}, V}$ is a regularly accretive extension of $F_{0}$, we have $u \in D\left(F_{\vec{a}, v}\right)$ and $F_{\vec{a}, v} u=H_{\vec{a}, v} u$. Therefore, $F_{\vec{a}, v}$ is an extension of $H_{\vec{a}, v}$ and $H_{\vec{a}, v}=F_{\vec{a}, v}$ for $F_{\vec{a}, V}$ and $H_{\vec{a}, V}$ are both self-adjoint. 


\section{ThE MAXIMAL SELF-ADJOINT REALISATION OF $P$}

Given the differential operator $P$, we can define a maximal realisation $\widetilde{H}_{\vec{a}, V}$ of $P$ in $L^{2}\left(R^{N}\right)$ as follows:

$$
\begin{gathered}
D\left(\widetilde{H}_{\vec{a}, V}\right)=\left\{u \in L^{2}\left(R^{N}\right):\left(\partial_{j}-i a_{j}\right) u \in L_{\text {loc }}^{2}\left(R^{N}\right),\right. \\
\left.|V|^{1 / 2} u \in L_{\text {loc }}^{2}\left(R^{N}\right), P u \in L^{2}\left(R^{N}\right)\right\}, \\
\widetilde{H}_{\vec{a}, V} u=P u, \quad u \in D\left(\widetilde{H}_{\vec{a}, V}\right),
\end{gathered}
$$

where $P$ acts on $u$ in the distribution sense. It is clear that $\widetilde{H}_{\vec{a}, V}$ is the extension of $H_{\vec{a}, V}$ obtained in Section 1. In fact, we have

THEOREM 3.1. $\widetilde{H}_{\vec{a}, V}=H_{\vec{a}, V}$.

CoRollary 3.2. $H_{\vec{a}, V}$ is the only self-adjoint realisation of $P$ in $L^{2}\left(R^{N}\right)$.

From (1.3) and $\left(H_{\vec{a}, V} u, v\right)=\sum_{j=1}^{N}\left(\left(\partial_{j}-i a_{j}\right) u,\left(\partial_{j}-i a_{j}\right) v\right)+\int_{R^{N}} V u \bar{v} d x$ for $u, v \in C_{0}^{\infty}\left(R^{N}\right)$, we can easily find $k>0$ such that

$$
\begin{aligned}
k\|\varphi\|^{2} & +\sum_{j=1}^{N} \int_{R^{N}}\left|\left(\partial_{j}-i a_{j}\right) \varphi\right|^{2} d x+\int_{R^{N}}|V| \cdot|\varphi|^{2} d x \geqslant\left(\left(H_{\vec{a}, V}+k\right) \varphi, \varphi\right) \\
& \geqslant\|\varphi\|^{2}+\frac{1-C_{1}}{2}\left(\int_{R^{N}} \sum_{j=1}^{N}\left|\left(\partial_{j}-i a_{j}\right) \varphi\right|^{2} d x+\int_{R^{N}}|V| \cdot|\varphi|^{2} d x\right)
\end{aligned}
$$

for all $\varphi \in C_{0}^{\infty}\left(R^{N}\right)$. Thus, we may define a norm on $C_{0}^{\infty}\left(R^{N}\right)$ as follow:

$$
\|\varphi\|_{1}=\left(\left(H_{\vec{a}, V}+k\right) \varphi, \varphi\right)^{1 / 2} .
$$

By completing $C_{0}^{\infty}\left(R^{N}\right)$ in the norm $\|\cdot\|_{1}$, we obtain a Hilbert space which we denote by $M$. From $[9$, Theorem 2.1], we have

$$
M=\left\{u \in L^{2}\left(R^{N}\right):\left(\partial_{j}-i a_{j}\right) u \in L^{2}\left(R^{N}\right),|V|^{1 / 2} u \in L^{2}\left(R^{N}\right)\right\} .
$$

For the proof of the Theorem 3.1, we need

Lemma 3.3. If there is a $k^{\prime}>0$ such that for all $\varphi \in C_{0}^{\infty}\left(R^{N}\right)$,

$$
\sum_{j=1}^{N}\left\|\left(\partial_{j}-i a_{j}\right) \varphi\right\|^{2}+\int_{R^{N}} V|\varphi|^{2} d x+k\|\varphi\|^{2} \geqslant k^{\prime}\|\varphi\|^{2},
$$


where $k$ is as in (3.1). Then the map $u \rightarrow(P+k) u$ is an injective map from $D\left(\widetilde{H}_{\vec{a}, V}+k\right)$ into $L^{2}\left(R^{N}\right)$.

Proof: Suppose $u \in D\left(\widetilde{H}_{\vec{a}, V}+k\right)$ such that $\left(\widetilde{H}_{\vec{a}, V}+k\right) u=0$. For any $\varepsilon>0$, define $u_{\varepsilon}=u /(1+\varepsilon|u|)$, then we have

(i) $u_{e} \in L_{\mathrm{loc}}^{2}\left(R^{N}\right),\left(\partial_{j}-i a_{j}\right) u_{e} \in L_{\mathrm{loc}}^{2}\left(R^{N}\right)$,

(ii) $u_{\varepsilon} \rightarrow u,\left(\partial_{j}-i a_{j}\right) u_{\varepsilon} \rightarrow\left(\partial_{j}-i a_{j}\right) u$ in $L_{\mathrm{loc}}^{2}\left(R^{N}\right)$ as $\varepsilon \rightarrow 0$.

In fact, $u_{e} \in L_{\text {loc }}^{2}\left(R^{N}\right)$ is obvious, and by $\left|u_{\varepsilon}-u\right|=\left(\varepsilon|u|^{2}\right) /(1+\varepsilon|u|) \leqslant|u|$ and the dominated convergence theorem, we obtain $u_{\varepsilon} \rightarrow u$ as $\varepsilon \rightarrow 0$ in $L_{\text {loc }}^{2}\left(R^{N}\right)$. Also since $u \in L^{2}\left(R^{N}\right) \subset L_{\text {loc }}^{1}\left(R^{N}\right), a_{j} u \in L_{\text {loc }}^{1}\left(R^{N}\right), 1 \leqslant j \leqslant N$, we can deduce $\partial_{j} u \in L_{\mathrm{loc}}^{1}\left(R^{N}\right)$ and $D\left(\widetilde{H}_{\vec{a}, V}\right) \subset H_{\mathrm{loc}}^{1,1}\left(R^{N}\right)$. By (1.8) we have $\partial_{j}|u| \in L_{\mathrm{loc}}^{2}\left(R^{N}\right)$. For any $\varphi$ in $C_{0}^{\infty}\left(R^{N}\right)$,

$$
\partial_{j}\left|u_{e} \varphi\right|=u_{\varepsilon}\left(\partial_{j} \varphi\right)+\varphi \cdot \frac{\partial_{j} u-\varepsilon u_{\varepsilon} \partial_{j}|u|}{1+\varepsilon|u|}
$$

This implies

$$
\begin{aligned}
& \left|\left(\partial_{j}-i a_{j}\right)\left(u_{\varepsilon} \varphi\right)-\left(\partial_{j}-i a_{j}\right)(u \varphi)\right| \\
& \quad=\left|\left(u_{\varepsilon}-u\right)\left(\partial_{j} \varphi\right)+\varphi \cdot \frac{-\varepsilon u_{\varepsilon} \partial_{j}|u|-\varepsilon|u|\left(\partial_{j}-i a_{j}\right) u}{1+\varepsilon|u|}\right| \\
& \quad \leqslant\left|\left(\partial_{j} \varphi\right) u\right|+\left|\left(\partial_{j}-i a_{j}\right) u\right| \cdot|\varphi| \in L^{2}\left(R^{N}\right),
\end{aligned}
$$

therefore $\left(\partial_{j}-i a_{j}\right)\left(u_{e} \varphi\right) \in L^{2}\left(R^{N}\right)$. Using (3.3) and the dominated convergence theorem, we obtain $\left(\partial_{j}-i a_{j}\right) u_{e} \rightarrow\left(\partial_{j}-i a_{j}\right) u$ as $\varepsilon \rightarrow 0$ in $L_{\text {loc }}^{2}\left(R^{N}\right)$. So we have proved (i) and (ii).

For any real function $\varphi \in C_{0}^{\infty}\left(R^{N}\right), u_{e} \varphi^{2} \in M \cap L^{\infty}\left(R^{N}\right)$. By (3.2), we have

$$
\sum_{j=1}^{N}\left(\left(\partial_{j}-i a_{j}\right) u,\left(\partial_{j}-i a_{j}\right)\left(u_{\varepsilon} \varphi^{2}\right)\right)+\int_{R^{N}}(V+k) u \bar{u}_{\varepsilon} \varphi^{2} d x=0 .
$$

Then

$$
\begin{aligned}
\int_{R^{N}} & \left(\left(\partial_{j}-i a_{j}\right) u \cdot \overline{\left(\partial_{j}-i a_{j}\right)\left(u_{e} \varphi^{2}\right)}+V\left|u_{e}\right|^{2} \varphi^{2}\right) d x+\int_{R^{N}} k\left|u_{e}\right|^{2} \varphi^{2} d x \\
= & \int_{R^{N}} k\left(u_{\varepsilon}-u\right) \bar{u}_{e} \varphi^{2} d x \\
& +\int_{R^{N}}\left(\left(\partial_{j}-i a_{j}\right)\left(u_{e}-u\right) \cdot \overline{\left(\partial_{j}-i a_{j}\right)\left(u_{e} \varphi^{2}\right)}-V\left(u-u_{e}\right) \bar{u} \varphi^{2}\right) d x \\
= & : I_{e}
\end{aligned}
$$


Since $\left(u-u_{e}\right) \bar{u}_{\varepsilon}\left(=\left(\varepsilon|u|^{3}\right) /\left((1+\varepsilon|u|)^{2}\right) \geqslant 0\right)$ is real, $\lim _{\varepsilon \rightarrow 0} \int_{R^{N}}\left(u_{\varepsilon}-u\right) \bar{u}_{\varepsilon} \varphi^{2} d x=0$ and $\lim _{e \rightarrow 0} \int_{R^{N}}\left(\partial_{j}-i a_{j}\right)\left(u_{e}-u\right) \cdot \overline{\left(\partial_{j}-i a_{j}\right)\left(u_{e} \varphi^{2}\right)} d x=0$, we have $\lim _{e \rightarrow 0} \operatorname{Im} I_{e}=0$.

By $\left(u-u_{\varepsilon}\right) \bar{u}_{e} \geqslant 0$, we have

$$
\begin{aligned}
\operatorname{Re} I_{\varepsilon}= & \operatorname{Re}\left(\int_{R^{N}}\left(\partial_{j}-i a_{j}\right)\left(u_{\varepsilon}-u\right) \cdot \overline{\left(\partial_{j}-i a_{j}\right)\left(u_{\varepsilon} \varphi^{2}\right)} d x+k \int_{R^{N}}\left(u_{\varepsilon}-u\right) \bar{u}_{\varepsilon} \varphi^{2} d x\right) \\
& \quad-\int_{R^{N}} V\left(u-u_{\varepsilon}\right) \bar{u}_{\varepsilon} \varphi^{2} d x \\
\leqslant & \operatorname{Re}(\cdots)+\int_{R^{N}} V_{-}\left(u-u_{\varepsilon}\right) \bar{u}_{\varepsilon} \varphi^{2} d x \\
\leqslant & \operatorname{Re}(\cdots)+\left(\int_{R^{N}} V_{-}\left|u-u_{\varepsilon}\right|^{2} \varphi^{2} d x\right)^{1 / 2}\left(\int_{R^{N}} V_{-} \varphi^{2}\left|u_{\varepsilon}\right|^{2} d x\right)^{1 / 2} .
\end{aligned}
$$

So from (i) and (ii), $\varlimsup_{\varepsilon \rightarrow 0}\left(\operatorname{Re} I_{e}+\operatorname{Im} I_{e}\right) \leqslant 0$. Also, since

$$
\begin{aligned}
& \left(\partial_{j}-i a_{j}\right) u_{\varepsilon} \cdot \overline{\left(\partial_{j}-i a_{j}\right)\left(u_{e} \varphi^{2}\right)} \\
& \quad=\sum_{j=1}^{N}\left|\left(\partial_{j}-i a_{j}\right)\left(u_{\varepsilon} \varphi\right)\right|^{2}-\left|u_{\varepsilon}\right|^{2} \cdot|\nabla \varphi|^{2}+2 i \operatorname{Im}\left(\sum_{j=1}^{N} \bar{u}_{e} \varphi \partial_{j} \varphi \cdot\left(\partial_{j}-i a_{j}\right) u_{e}\right)
\end{aligned}
$$

we have

$$
\begin{aligned}
& \int_{R^{N}}\left(\sum_{j=1}^{N}\left|\left(\partial_{j}-i a_{j}\right)\left(u_{\varepsilon} \varphi\right)\right|^{2}-\left|u_{\varepsilon}\right|^{2}|\nabla \varphi|^{2}\right. \\
& \left.\quad+2 i \operatorname{Im} \sum_{j=1}^{N}\left(\bar{u}_{\varepsilon} \varphi \cdot \partial_{j} \varphi \cdot\left(\partial_{j}-i a_{j}\right) u_{\varepsilon}\right)+V\left|u_{\varepsilon}\right|^{2} \varphi^{2}+k\left|u_{e}\right|^{2} \varphi^{2}\right) d x \equiv I_{\varepsilon} .
\end{aligned}
$$

By $\lim _{e \rightarrow 0} \operatorname{Im} I_{e}=0$,

$$
\lim _{\varepsilon \rightarrow 0}\left|\operatorname{Im} \sum_{j=1}^{N} \int_{R^{N}} \bar{u}_{\varepsilon} \varphi \partial_{j} \varphi \cdot\left(\partial_{j}-i a_{j}\right) u_{\varepsilon} d x\right|=0
$$

Then from (3.2) and the condition, we have

$$
\sum_{j=1}^{N}\left\|\left(\partial_{j}-i a_{j}\right)\left(u_{\varepsilon} \varphi\right)\right\|^{2}+\int_{R^{N}} V\left|u_{\varepsilon} \varphi\right|^{2} d x+\int_{R^{N}} k\left|u_{\varepsilon} \varphi\right|^{2} d x \geqslant k^{\prime}\left\|u_{\varepsilon} \varphi\right\|^{2}
$$


and

$$
\begin{aligned}
0 & \geqslant \varlimsup_{\varepsilon \rightarrow 0}\left(\operatorname{Re} I_{e}+\operatorname{Im} I_{e}\right) \\
& =\varlimsup_{e \rightarrow 0} \operatorname{Re} \int_{R^{N}}\left(\sum_{j=1}^{N}\left|\left(\partial_{j}-i a_{j}\right)\left(u_{\varepsilon} \varphi\right)\right|^{2}-\left|u_{e}\right|^{2}|\nabla \varphi|^{2}+k\left|u_{\varepsilon}\right|^{2} \varphi^{2}+V\left|u_{\varepsilon} \varphi\right|^{2}\right) d x \\
& \geqslant \varlimsup_{\varepsilon \rightarrow 0}\left(k^{\prime}\left\|u_{e} \varphi\right\|^{2}-\int_{R^{N}}\left|u_{e}\right|^{2}|\nabla \varphi|^{2} d x\right) \\
& =k^{\prime}\|u \varphi\|^{2}-\int_{R^{N}}|u|^{2}|\nabla \varphi|^{2} d x,
\end{aligned}
$$

that is, $k^{\prime}\|u \varphi\|^{2} \leqslant \int_{R^{N}}|u|^{2}|\nabla \varphi|^{2} d x$. Taking $\varphi_{\varepsilon}(x)=\Psi(x / \varepsilon)$, where $\Psi \in C_{0}^{\infty}\left(R^{N}\right)$, $\Psi(x)=1$ when $|x| \leqslant 1 ; \Psi(x)=0$ when $|x| \geqslant 2$ and $0 \leqslant \Psi \leqslant 1,\left|\partial_{j} \Psi(y / \varepsilon)\right|=$ $o(1 / \varepsilon)(\varepsilon \rightarrow \infty)$, we have $k^{\prime}\|u\| \leqslant 0$ and $u \equiv 0$.

Proof of Theorem 3.1: $\left(H_{\vec{a}, V}+k\right)^{-1}$ is a bounded linear operator in $L^{2}\left(R^{N}\right)$ for suitable $k>0$. Suppose $u \in D\left(\widetilde{H}_{\vec{a}, V}+k\right), v=u-\left(H_{\vec{a}, V}+k\right)^{-1}\left(\left(\widetilde{H}_{\vec{a}, V}+k\right) u\right)$. Since $D\left(H_{\vec{a}, V}\right) \subset D\left(\widetilde{H}_{\vec{a}, V}\right)$, we have $v \in D\left(\tilde{H}_{\vec{a}, V}+k\right)$. Also since $(P+k) v=0$ and from (3.1), we have $v \equiv 0$ by Lemma 3.3. So $u \in D\left(H_{\vec{a}, V}+k\right)$ and $\widetilde{H}_{\vec{a}_{,},}=$ $\boldsymbol{H}_{\vec{a}, V}$.

\section{The essential SElf-adjointness of $P$ on $C_{0}^{\infty}\left(R^{N}\right)$}

In this section, we consider the essential self-adjoint extension of the Schrödinger operator $P=-\sum_{j=1}^{N}\left(\partial_{j}-i a_{j}\right)^{2}+V$, where $\vec{a} \in L_{\text {loc }}^{4}\left(R^{N}\right)^{N}$, div $\vec{a} \in L_{\text {loc }}^{2}\left(R^{N}\right), V=$ $V_{1}+V_{2}, V_{i} \in L_{\text {loc }}^{2}\left(R^{N}\right), i=1,2, V_{1}(x) \geqslant-C|x|^{2}(C \geqslant 0), 0 \geqslant V_{2} \in K_{N}$.

First, we prove the following result.

LEMma 4.1. Let $\vec{a}, V$ be as above. Then there exist constants $C_{3}>0, C_{4}>0$ such that for all $u \in C_{0}^{\infty}\left(R^{N}\right)$,

$$
\sum_{j=1}^{N} \int_{B_{m}}\left|\left(\partial_{j}-i a_{j}\right) u\right|^{2} d x \leqslant C_{3} \int_{B_{m}}\left|P_{u}\right|^{2} d x+C_{4} m^{2} \int_{B_{m}}|u|^{2} d x
$$

where $B_{m}=\left\{x \in R^{N}: m / 2 \leqslant|x| \leqslant 3 m\right\}, m>0$.

Proof: Take $\xi \in C_{0}^{\infty}\left(R^{N}\right), 0 \leqslant \xi \leqslant 1, \xi(x)=1$ when $1 \leqslant|x| \leqslant 2 ; \xi(x)=0$ when $|x| \geqslant 3$ or $|x| \leqslant 1 / 2$. For any positive integer $m, \xi_{m}=\xi(x / m)$. By $V_{2} \in K_{N}$, 
for any $\varepsilon>0$, there exists $M\left(\varepsilon, V_{2}\right)>0$ such that

$$
\left|\left(V_{2} \xi_{m} u, \xi_{m} u\right)\right| \leqslant \varepsilon \int_{R^{N}}\left|\nabla\left(\xi_{m} u\right)\right|^{2} d x+M\left(\varepsilon, V_{2}\right) \int_{R^{N}}\left|\xi_{m} u\right|^{2} d x
$$

for $u \in C_{0}^{\infty}\left(R^{N}\right)$. Set $K=\max _{y \in R^{N}}|\nabla \xi(y)|$, then $\left|\nabla \xi_{m}(x)\right| \leqslant K / m$ and

$$
\left|\partial_{j}\left(\xi_{m} u\right)\right|^{2} \leqslant 2\left(\partial_{j} \xi_{m}\right)^{2}|u|^{2}+2 \xi_{m}^{2}\left|\partial_{j} u\right|^{2} .
$$

Therefore, there exists a constant $C_{\varepsilon}>0$ such that for any $u \in C_{0}^{\infty}\left(R^{N}\right)$.

$$
\left|\left(V_{2} u, \xi_{m}^{2} u\right)\right| \leqslant 2 \varepsilon \int_{R^{N}} \xi_{m}^{2}|\nabla| u \|^{2} d x+C_{e} \int_{R^{N}}|u|^{2} d x .
$$

Taking $\varepsilon=1 / 16$, we have

$$
\begin{aligned}
\left|\left(P u, \xi_{m}^{2} u\right)\right|= & \left|\left((\nabla-i \vec{a}) u,(\nabla-i \vec{a})\left(\xi_{m}^{2} u\right)\right)+\left(V u, \xi_{m}^{2} u\right)\right| \\
\geqslant & \left|\sum_{j=1}^{N}\left(\left(\partial_{j}-i a_{j}\right) u,\left(\partial_{j}-i a_{j}\right)\left(\xi_{m}^{2} u\right)\right)\right|-C(3 m)^{2} \int_{B_{m}}|u|^{2} d x \\
& -\frac{1}{8} \int_{B_{m}}|\nabla| u \|^{2} d x-C_{1 / 16} \int_{B_{m}}|u|^{2} d x .
\end{aligned}
$$

Also since

$$
\begin{aligned}
& \sum_{j=1}^{N}\left(\left(\partial_{j}-i a_{j}\right) u,\left(\partial_{j}-i a_{j}\right)\left(\xi_{m}^{2} u\right)\right) \\
& \quad=\sum_{j=1}^{N}\left(\left(\partial_{j}-i a_{j}\right) u, \xi_{m}^{2}\left(\partial_{j}-i a_{j}\right) u\right)+\sum_{j=1}^{N}\left(\left(\partial_{j}-i a_{j}\right) u, 2 \xi_{m}\left(\partial_{j} \xi_{m}\right) u\right),
\end{aligned}
$$

we have

$$
\begin{aligned}
\left|\left(P u, \xi_{m}^{2} u\right)\right| \geqslant & \sum_{j=1}^{N} \int_{B_{m}}\left|\left(\partial_{j}-i a_{j}\right) u\right|^{2} d x-\frac{1}{4} \sum_{j=1}^{N} \int_{B_{m}}\left|\left(\partial_{j}-i a_{j}\right) u\right|^{2} d x \\
& -4 \int_{B_{m}}|u|^{2}\left|\nabla \xi_{m}\right|^{2} d x-C(3 m)^{2} \int_{B_{m}}|u|^{2} d x \\
& -\frac{1}{8} \int_{B_{m}}|\nabla| u \|^{2} d x-C_{1 / 16} \int_{B_{m}}|u|^{2} d x
\end{aligned}
$$

This implies that

$$
\sum_{j=1}^{N} \int_{B_{m}}\left|\left(\partial_{j}-i a_{j}\right) u\right|^{2} d x \leqslant C_{3}^{\prime} \int_{B_{m}}|P u|^{2} d x+C_{4}^{\prime} m^{2} \int_{B_{m}}|u|^{2} d x+\frac{1}{8} \int_{B_{m}}|\nabla| u \|^{2} d x
$$


for suitable constants $C_{3}^{\prime}, C_{4}^{\prime}>0$. Also by (1.8), we have

$$
\sum_{j=1}^{N} \int_{B_{m}}\left|\left(\partial_{j}-i a_{j}\right) u\right|^{2} d x \leqslant C_{3} \int_{B_{m}}|P u|^{2} d x+C_{4} m^{2} \int_{B_{m}}|u|^{2} d x
$$

for suitable constants $C_{3}, C_{4}>0$.

Theorem 4.2. Let $\vec{a}, V$ be as above, then $P=-\sum_{j=1}^{N}\left(\partial_{j}-i a_{j}\right)^{2}+V$ is essential self-adjoint on $C_{0}^{\infty}\left(R^{N}\right)$.

Proof: Since $P$ is symmetric, if we want to prove that $P$ is essential self-adjoint on $C_{0}^{\infty}\left(R^{N}\right)$, we only need to prove that for any $\varphi \in C_{0}^{\infty}\left(R^{N}\right)$, if $f \in L^{2}\left(R^{N}\right)$, $(f, P \varphi)=0$, then $f \equiv 0$. Thus, in the sequel, we suppose $f \in L^{2}\left(R^{N}\right),(f, P \varphi)=0$ for any $\varphi \in C_{0}^{\infty}\left(R^{N}\right)$.

If $V$ satisfies (H), put

$$
V_{1}^{(k)}(x)= \begin{cases}V_{1}(x) & |x| \leqslant k \\ -C k^{2} & |x|>k\end{cases}
$$

Then $\left(V_{1}^{(k)}\right)_{-}$is a bounded function. By the discussion in Section 1 , we have $P_{k}:=$ $-\sum_{j=1}^{N}\left(\partial_{j}-i a_{j}\right)^{2}+V_{2}+V_{1}^{(k)}$ is essential self-adjoint on $C_{0}^{\infty}\left(R^{N}\right)$ in the sense of form and we denote the self-adjoint realisation of $P_{k}$ by $\bar{P}_{k}$. Moreover, by (4.2) we have

$$
\left|\left(\bar{P}_{k} u, \xi_{m}^{2} u\right)\right|=\left|\left((\nabla-i \vec{a}) u,(\nabla-i \vec{a})\left(\xi_{m}^{2} u\right)\right)+\left(\left(V_{2}+V_{1}^{(k)}\right) u, \xi_{m}^{2} u\right)\right|
$$

for $u \in D\left(\bar{P}_{k}\right)$. Using the same methods in the proof of Lemma 4.1, we have there exist constants $C_{5}, C_{6}>0$ such that

$$
\sum_{j=1}^{N} \int_{B_{m}}\left|\left(\partial_{j}-i a_{j}\right) u\right|^{2} d x \leqslant C_{5} \int_{B_{m}}\left|\bar{P}_{k} u\right|^{2} d x+C_{6} m^{2} \int_{B_{m}}|u|^{2} d x .
$$

Take $\eta \in C_{0}^{\infty}\left(R^{N}\right), \eta(x)=1$ for $|x| \leqslant 1 ; \eta(x)=0$ for $|x| \geqslant 2$ and set $\eta_{m}(x)=$ $\eta(x / m)$. For any $u \in C_{0}^{\infty}\left(R^{N}\right)$,

$$
(P+i)\left(u \eta_{m}\right)=\eta_{m}(P+i)-2 \nabla \eta_{m} \cdot \vec{D} u-\left(\Delta \eta_{m}\right) u
$$

where $\vec{D}=\left(\partial_{1}-i a_{1}, \partial_{2}-i a_{2}, \cdots, \partial_{N}-i a_{N}\right)$. From this, we have

$$
\left(f, \eta_{m}(T+i) u\right)=\left(f,\left(\Delta \eta_{m}\right) u\right)+2\left(f, \nabla \eta_{m} \cdot \vec{D}_{u}\right)
$$


and for any $u \in C_{0}^{\infty}\left(R^{N}\right), k \geqslant 3 m$,

$$
\eta_{m}(T+i) u=\eta_{m}\left(P_{k}+i\right) u
$$

Taking $k=3 m$, we have

$$
\left(f \eta_{m},\left(P_{3_{m}}+i\right) u\right)=\left(f,\left(\Delta \eta_{m}\right) u\right)+2\left(f, \nabla \eta_{m} \cdot \vec{D}_{u}\right) .
$$

Since $\bar{P}_{3 m}$ is essential self-adjoint on $C_{0}^{\infty}\left(R^{N}\right)$ in the sense of form, (4.4) also holds for $u \in D\left(\bar{P}_{k}\right)$. Therefore there exists $u_{m} \in D\left(\bar{P}_{3 m}\right)$ such that $\left(\bar{P}_{3_{m}}+i\right) u_{m}=f \eta_{m}$. So, we have

$$
\begin{aligned}
\left\||f|^{2} \eta_{m}^{2}\right\|^{2} & =\left\|\left(\bar{P}_{3 m}+i\right) u_{m}\right\|^{2}=\left(f,\left(\Delta \eta_{m}\right) u_{m}\right)+2\left(f, \nabla \eta_{m} \cdot \vec{D} u_{m}\right) \\
& \leqslant\|f\|_{L^{2}\left(B_{m}\right)}\left(M m^{-2}\left\|u_{m}\right\|+2 M m^{-1}\left\|\left|\vec{D} u_{m}\right|\right\|_{L^{2}\left(B_{m}\right)}\right)
\end{aligned}
$$

for suitable constant $M>0$. Since $\bar{P}_{3 m}$ is a self-adjoint operator and $\left(\bar{P}_{3 m}+i\right) u_{m}=$ $f \eta_{m}$, we have $\left\|u_{m}\right\| \leqslant\left\|f \eta_{m}\right\| \leqslant\|f\|$. Also by (4.3),

$$
\left\|\left|\vec{D} u_{m}\right|\right\|_{L^{2}\left(B_{m}\right)}^{2} \leqslant C_{5} \int_{B_{m}}\left|\left(\bar{P}_{3 m}+i\right) u_{m}\right|^{2} d x+C_{6} m^{2} \int_{B_{m}}\left|u_{m}\right|^{2} d x .
$$

So from (4.5) and (4.6), there exists $C_{7}>0$ such that

$$
\left.|| f\right|^{2} \eta_{m}^{2}\left\|^{2} \leqslant C_{7}\right\| f \|_{L^{2}\left(B_{m}\right)}
$$

Let $m \rightarrow \infty$, then we have $\left\||f|^{2}\right\|=0$; thus $f \equiv 0$.

\section{REFERENCES}

[1] M. Aizenman and B. Simon, 'Brownian motion and Harnack's inequality for Schrödinger operators', Comm. Pure Appl. Math. 35 (1982), 209-273.

[2] A.M. Hinz and G. Stolz, 'Polynomial boundedness of eigensolutions and the spectrum of Schrödinger operator', Math. Ann. 294 (1992), 195-211.

[3] T. Kato, Perturbation theory for linear operators, 2nd ed. (Springer-Verlag, Berlin, Heidelberg, New York, 1966).

[4] H. Leinfelder and C. Simader, 'Schrödinger operators with singular magnetic vector potentials', Math. Z. 196 (1981), 1-19.

[5] M. Reed and B. Simon, Methods of modern mathematical physics, IV. Analysis of operators (Academic Press, London, 1978). 
[6] M. Schechter, Spectra of partial differential operators (North-Holland, Amsterdam, New York, Oxford, 1986).

[7] C.G. Simader, 'Remarks on essential self-adjoint of Schrödinger operators with singular electrostatic potentials', J. Reine Angew. Math. 431 (1992), 1-6.

[8] C.G. Simader, 'A elementary proof of Harnack's inequality for Schrödinger operators and related topics', Math. Z. 203 (1990), 129-152.

[9] B. Simon, 'Maximal and minimal Schrödinger forms', J. Opt. Theory 1 (1979), 37-47.

Department of Mathematics, Physics and Mechanics Nanjing University of Aeronautics and Astronautics Nanjing 210016

People's Republic of China 